\title{
Battery Ingestion: A Potentially Life-Threatening Issue
}

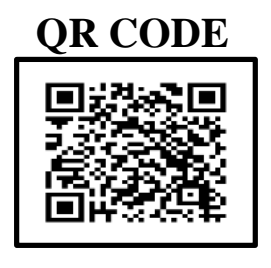

\section{AMOS MASIH}

Button battery ingestion cases are rare but possibilities of these turning into life-threatening or fatal conditions are high. Such an unfortunate incidence may progress to serious complications and require major surgery if the foreign body is not identified or removed well in time. Lithium is a possible component of button batteries being used these days. The consequences of batteries containing lithium are medically significant. This review is to focus on general safety information regarding button battery ingestion, facts learned from reports published previously, and conclusion derived from brilliant literature.

KEYWORDS: Button Batteries, Lithium, Ingestion

\section{INTRODUCTION}

Almost all the physicians during their career encounter cases of foreign body ingestion. In most of these cases, the concerned patients are children with an age range of six months to three years., ${ }^{1,2}$ The yearly rate of occurrences of button battery ingestion is approximately around ten in a million people. Button batteries can be found in wrist watches, calculators, hearing aids, remote control devices, and many toys. Easy accessibility of these small sized batteries to infants and children may pose to a serious health hazard.

With an escalating rise in technological development, electronic toys and gadgets have come up in a ubiquitous manner making a part of every household. In earlier times, when female workforce was minimal and housewives used to take care of kids, chances of a child ingesting such items was less. Trend has also changed from joint families to nuclear ones, with small children playing alone or being taken care by paid babysitters, leading to increase in instances of foreign body ingestion. The ingestion of these batteries is commonly unintentional and accidental but lack of cognitive development at small age may also lead to ingestion following fascination. Chances of foreign body ingestion may be more in mentally subnormal children.

Kalyanshettar SS et al. reported a case of 8 year old subnormal male who ingested a $3 \mathrm{~V}$ lithium button battery. ${ }^{3}$ Due to scarcity of research work and longterm studies, treatment strategies are mostly derived from professional opinion or relevant experience. Back in 1977, Blatnik DS et al. reported the first case of button battery ingestion in a child who ingested a camera battery, which got impacted in the proximal esophageal region. ${ }^{4}$ The button batteries may contain lithium, mercury, silver or a hydroxide compound of sodium or potassium. ${ }^{5}$ Although the potential of lithium batteries is 3 volts which is double than 1.5 volts for other button batteries, if ingested they can navigate and clear the gastrointestinal tract due to their more slender shape. ${ }^{6}$ The concerned doctor should thoroughly investigate the case of foreign body ingestion as around 30\% of children with foreign body in esophageal tract will not show any symptoms. ${ }^{7}$ Differential diagnosis of foreign body ingestion should always be kept in mind in case a child subject shows up with symptoms of chest pain and proper history along with radiographs should be recorded to rule out the condition. In $60-70 \%$ of the cases, coin shaped objects are found lodged in region of upper esophageal sphincter, $10-20 \%$ at the level of aortic notch in mid esophagus and $20 \%$ above the lower esophageal sphincter. ${ }^{8}$

The most common symptoms include chest pain, abdominal pain, restlessness, irritability, vomiting, drooling, melena, hematemesis, inability to swallow, cough, wheezing, stridor, and choking. Injurious complications of button battery in gastrointestinal tract may be due to triggering of an electrolytic current, hydrolyzation of tissue fluids, hydroxide generation and leakage and absorption of battery contents leading to low voltage burns of mucosa, esophageal perforations, pressure necrosis, strictures, temporary or permanent paralysis of vocal chords, tracheoesophageal fistula, major bleeding, 
mediastinitis, aortic perforation, abrasions, lacerations, pulmonary edema, tracheal stenosis, aspiration pneumonia, empyema, abscess, pneumothorax, spondylodiscitis and esophageal diverticulum \& death in some cases. ${ }^{5-9,10}$

The mucosal injury from button batteries may start within an hour of contact and entangle all the layers of the esophageal in 4 hours. ${ }^{7}$ The chances of developing serious complications and requiring major surgeries like thoracotomy, removal of foreign body with esophagostomy and esophagoplasty are less if the foreign body is identified and removed without any kind of delay.

The associated risk factors include large size of the battery more, small age less group, chemical contents of the battery, life of battery and ingestion of battery along with magnet. ${ }^{11-15}$

Following facts have also been derived from literature: A vast horizon of suspicion is important as many cases don't report any symptoms, investigations like chest and abdomen radiography can help in confirmation of the diagnosis and site location of the foreign body, vomiting induction should not be tried to avoid risk of regurgitation and lethal airway obstruction, blood or urine assays do not help much, due to a high potential of lithium batteries they should be removed within two hours of ingestion to avoid injurious complications, a few delayed complications may occur in 1-3 weeks of ingestion and fatal hemorrhage may occur within 12-14 hours of ingestion, oral antacids are found to be useful in decreasing corrosion caused due to ingested batteries and ingestions not witnessed and asymptomatic in nature may lead to misdiagnosis or delayed diagnosis. $^{11-18}$

Definite diagnosis is of prime importance in cases of button battery ingestion, as they can be mistaken for coins or some other objects on radiographs but are much more hazardous than them. ${ }^{19-21}$ Lateral radiographs and posterior-anterior views are good investigatory options as button batteries have a typical step-off sign appearance and a double-density or double-ring shadow on them respectively.,19,22-23 In asymptomatic patients, there is no requirement of a rabid treatment. Sometimes, it is safe to manage the case with a conservative approach. It is important to look for the anticipated signs and symptoms and then proceed with the appropriate intervention. As esophagus is the most vulnerable to button battery lodgment, most of the cases require urgent endoscopic intervention. The asymptomatic cases with battery present in stomach can be managed conveniently with stool examination and serial skiagrams at regular follow ups. Cases with vascular involvement due to erosion caused by battery contents can be fatal and these cases need prompt medical intervention. However, prevention is always better than cure and is also applicable to cases of button battery ingestion.

\section{CONCLUSION}

In the recent years, there has been an alarming escalation of button battery ingestion among young children across the globe because they are easily accessible. Button battery ingestion can result into life-threatening or fatal complications very swiftly. Thus, preventive measures are mandatory to avoid critical complications and death. Parents of young children should be educated about the potential hazards of button battery ingestion, so they become more cautious and put extra efforts in taking good care of their kids, not leaving them isolated and keeping a regular check on items which could be ingested by children. Tiny items and the ones that have any kind of chemical content should be kept in such places that cannot be reached by children. Children at certain age, parents and healthcare professionals need to be imparted knowledge regarding the hazards that may follow button battery ingestion. There is also a need to formulate better management plans and strategies to reduce the instances of butter battery ingestion. Early diagnosis, correct diagnosis and timely intervention are of paramount importance in treating a case of button battery ingestion.

\section{REFERENCES}

1. Cheng W, Tam PKH. Foreign-body ingestion in children: experience with 1,265 cases. Journal of Pediatric Surgery 1999;34(10):1472-76.

2. Litovitz T, Whitaker N, Clark L. Preventing battery ingestions: an analysis of 8648 cases. Pediatrics 2010;125(6):1178-83.

3. Kalyanshettar SS, Patil S, Upadhye G. Button battery ingestion-case report and review. J Clin Diagn Res. 2014;8:PDo1-PDo2.

4. Blatnik DS, Toohill RJ, Lehman RH. Fatal complication from an alkaline battery foreign body in the esophagus. Ann Otol Rhinol Laryngol 1977;86:6115. Banerjee R, Rao GV, Sriram PV, Reddy KS, 
Nageshwar Reddy D. Button battery ingestion. Indian J Pediatr 2005;72:173-4.

6. Litovitz TL. Battery ingestions: Product accessibility and clinical course. Pediatrics 1985;75:469-76.

7. Khan S, Orenstein SR. Foreign bodies in esophagus. Nelson textbook of pediatrics. 19th edition. Philadelphia: Elsevier; 2012: 1271.

8. Kay M, Wyllie R. Pediatric foreign bodies and their management. Curr Gastroenterol Rep. 2005;7:212-18.

9. Temple DM, McNeese MC. Hazards of battery ingestion. Pediatrics 1983;71:100-3.

10. Samad L, Ali M, Ramzi H. Button battery ingestion: Hazards of esophageal impaction. J Pediatr Surg 1999;34:1527-31.

11. Abdollahi Fakhim S, Bayazian G, Sohrabpour M. Neglected esophageal button battery ingestion: Local protocol for management. Egypt J Ear Nose Throat Allied Sci 2013;14:27-31.

12. Yamashlta M, Saito S, Koyama K, Hattori H, Ogata T. Esophageal electrochemical burns by button type alkaline batteries in dogs. Vet Hum Toxicol 1987;29:226-30.

13. Yoshikawa T, Asai S, Takekawa Y, Kida A, Ishikawa

$\mathrm{K}$. Experimental investigation of battery induced esophageal burns in rabbits. Crit Care Med 1997;25:2039-44.

14. Yasui T. Hazarous effects due to alkaline button battery ingestion: An experimental study. Ann Emerg Med 1986;15:901-6.
15. Langkau JF, Noesges RA. Esophageal burns from battery ingestion. Am J Emerg Med 1985;3:265.

16. Litovitz TL. Button battery ingestions. A review of 56 cases. JAMA 1983;249:2495-500.

17. Litovitz T, Butterfield AB, Holloway RR, Marion LI. Button battery ingestion: Assessment of therapeutic modalities and battery discharge state. J Pediatr 1984;105:868-73.

18. Gopal M, Westgarth-Taylor C, Loveland J. Repair of tracheo-oesophageal fistula secondary to button battery ingestion: a combined cervical and median sternotomy approach. Afr J Paediatr Surg. 2015; 12:9193.

19. Yardeni D, Yardeni H, Coran AG, et al. Severe esophageal damage due to button battery ingestion: can it be prevented? Pediatr Surg Int. 2004;20:496501.

20. Bernstein JM, Burrows SA, Saunders MW. Lodged oesophageal button battery masquerading as a coin: an unusual cause of bilateral vocal cord paralysis. Emerg Med J. 2007;24:e15.

21. Lin VY, Daniel SJ, Papsin BC. Button batteries in the ear, nose and upper aerodigestive tract. Int J Pediatr Otorhinolaryngol. 2004;68:473-479.

22. Fuentes S, Cano I, Benavent MI, et al. Severe esophageal injuries caused by accidental button battery ingestion in children. J Emerg Trauma Shock. 2014;7:316-321.

23. Gohil R, Culshaw J, Jackson P, et al. Accidental button battery ingestion presenting as croup. J Laryngol Otol. 2014;128:292-295. 